\title{
Research of Tire Pressure Monitoring System
}

\author{
Xu Yuan \\ School of North China Electric Power University, Baoding 071000, China \\ 15733266829@163.com
}

Key words: TPMS, Indirect TPMS, Direct TPMS

\begin{abstract}
Nowadays, the car has become an important means of transportation, the safety of the car has been paid more and more attention by people. The abnormal running will lead to flat tire, TPMS system for the timely monitoring of tire pressure, application of different types of TPMS system are different from each other. Based on the sensor types, monitoring methods and monitoring principle of TPMS system for the classification and different mathematical models are given for different TPMS systems. A more comprehensive definition of the structure of all types of TPMS, which will facilitate analysis and design of TPMS.
\end{abstract}

\section{Introduction}

Since the industrial age, the automobile has gradually become an important means of transportation. How to ensure traffic safety has been the focus of people's attention. After a lot of research and data accumulation, it summed up traffic safety have great relevance with the working conditions of tires. Therefore, the research on the tire pressure monitoring has become the focus of attention. [3]

Statistical data show that the accidents caused by tire puncture accounted for about $30 \%$ to $40 \%$ of highway accidents. Meanwhile, under pressure is also a significant risk of traffic accidents. Pressure is a important factor that leads to the change of tire performance, high pressure will decrease the elasticity of tire, reduce the contact area with the ground, and reduce the coefficient of friction. Eventually, high pressure will lead to the vehicle to skid, not smooth, bumps, flat tire, etc; but when the tire pressure is too low, not only will increase the driving resistance and fuel consumption, but also enable the tire a sharp rise in temperature, causing a flat tire. Obviously, to maintain proper tire pressure can not be ignored. ${ }^{[1]}$

Now TPMS (Tire Pressure Monitoring System) on the market is mainly divided into two types, the indirect TPMS (WSB TPMS) and the direct TPMS (PSB TPMS). ${ }^{[4]}$

\section{Indirect TPMS}

Indirect TPMS is using the ABS wheel speed sensor or other non-pressure sensor signal, calculating indirect tire pressure by the tire mechanics model and algorithm, or using the pressure differences between the tires to monitor tire pressure, there are three common monitoring method.

2.1 Frequency Analysis Method

This type monitor tire pressure by monitoring the vibration frequency related to tire pressure. The rubber tire will produce a similar vibration like the spring when automobile is running, and there exit a linear relationship between the elasticity coefficient and the tire pressure. Therefore, processing the signal generated by the ABS wheel speed sensor with VSC, the torsional vibration frequency of the tire can be obtained, then the elasticity coefficient of the tire can be obtained, and then the tire pressure can be calculated. Relation to $f=2 \pi \sqrt{m / k}$, the control flow chart is as follows: 


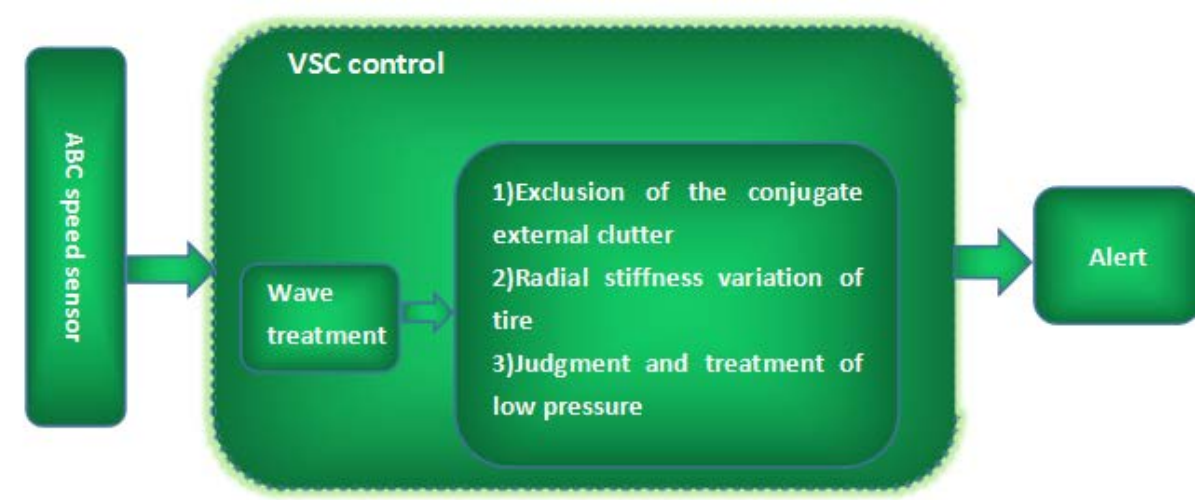

Figure 1: Vibration analysis TPMS working principle diagram

\subsection{Speed Analysis Method}

The tire radius is compared with the nonlinear change of the static wheel speed, and the difference is zero when the tire radius is close to the optimum value. The principle is: when the tire pressure drops, rolling radius of the tire will decrease, resulting in high speed than the other tires. By comparing the rotational speed of the tire between the tire pressure monitor to achieve the purpose.

Generally used for static nonlinear methods, such as:

$$
\gamma=\frac{w_{1}}{w_{2}}-\frac{w_{3}}{w_{4}}=\frac{R_{2}}{R_{1}}-\frac{R_{4}}{R_{3}}=\frac{R_{2} R_{3}-R_{1} R_{4}}{R_{1} R_{3}}
$$

The $w_{1}, w_{2}, w_{3}, w_{4}$ and $R_{1}, R_{2}, R_{3}, R_{4}$ respectively refers to the angular velocity and the effective radius of the left front wheel , right front wheel, rear left wheel and right rear wheel. When $\gamma$ is not zero, it indicates that a tire is under pressure. The disadvantage of this method is that it can not be detected in the case of two coaxial or the same side and four tire pressure drop at the same time and accuracy of the system is not high.

\subsection{Magnetic Detection Method}

The magnetic indirect TPMS system is composed of a pressure sensor, a Hall element and an electronic control unit. The tire pressure sensor is arranged on the wheel rim, and the Hall device is installed on the bracket of the suspension strut or the wheel brake bottom plate, which works as follows: When driving, a change in tire pressure causes a change in the pressure sensor element magnetic field direction, then the output signal of the Hall device $\mathrm{V}_{H}$ also change, thus achieving a non-contact pneumatic pressure signal transmitted by the tire to the vehicle body. The relationship is $\mathrm{V}_{\mathrm{H}}=\mathrm{K} \times \mathrm{B}, \mathrm{K}$ is the sensitivity of the Hall device and the system is as follows:

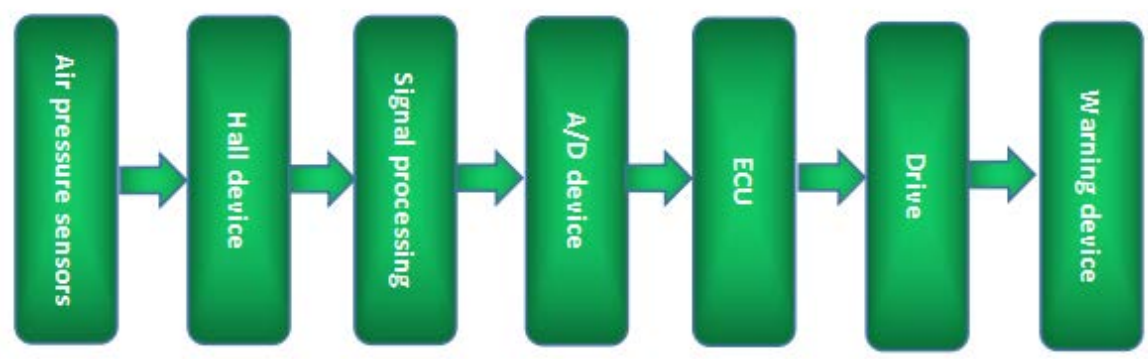

Figure 2: Magnetic indirect TPMS working principle diagram

\section{Direct Tire Pressure Monitoring System}

Direct TPMS monitor tire pressure and temperature by using pressure sensor and temperature sensor installed in each tire, and the tire pressure is displayed. Direct TPMS are better than indirect TPMS from the functionality and performance. Direct TPMS can be divided into the tire module and the central receiver module, the principle is as follows: 

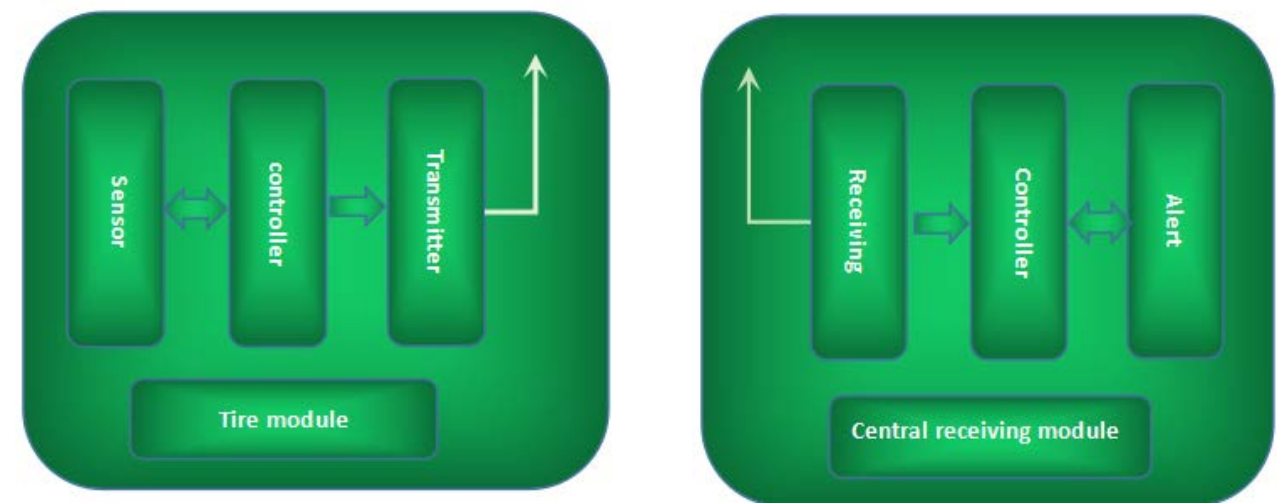

Figure 3: Block Diagram Of Direct TPMS

Direct TPMS can be divided into active type and passive type: Active TPMS, refers to the transmitter contains power module, is mainly composed of two parts, one part is the TPMS transmitter, the other part is the TPMS receiver. The pressure sensor which is provided with a lithium battery as the power source is used for the direct measurement of the tire pressure of the tire, and is transmitted to the monitor which is installed on the driver's desk through wireless modulation. When the tire pressure is too low, it will automatically alarm. The function block diagram of the transmitting module and receiving module are shown below:

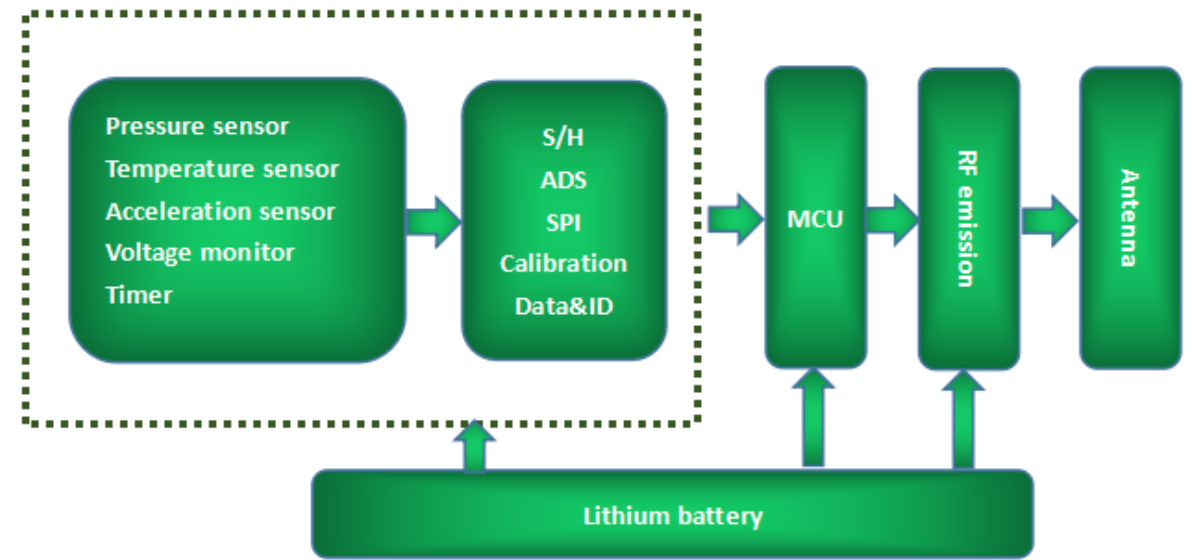

Figure 4: functional block diagram of the transmitting module.

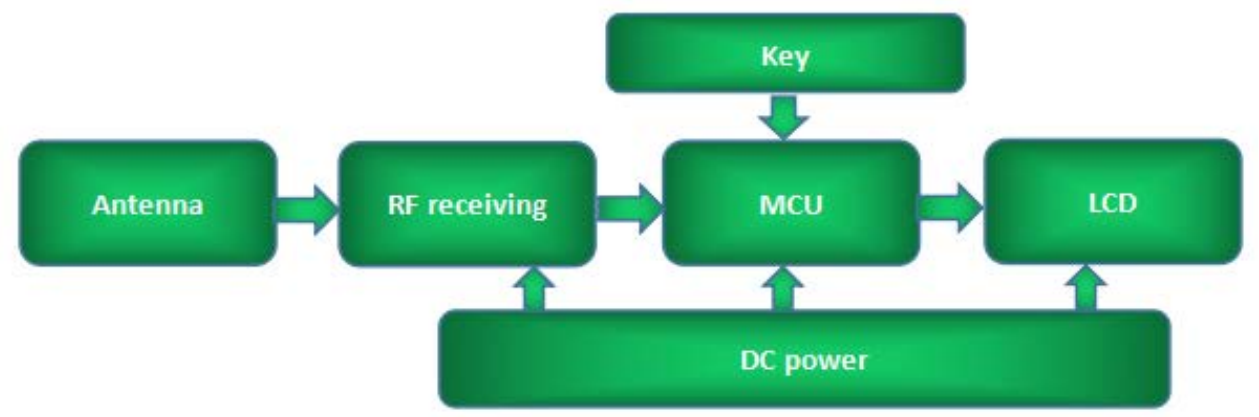

Figure 5: functional block diagram of the receiving module

The disadvantage of such monitoring system are:(1)The shorter the life due to limitations of lithium batteries(2)the quality of the sensor system is mostly in 20g, which will cause greater dynamic loads. (3)Because of the harsh working environment inside the tire, all the components and materials should meet the temperature range of -40 to +125 .

Passive TPMS is also called a TPMS without a battery, that is, inside the tire sensor does not use batteries. There are two directions: energy sources are transmit power from RFID read heads or mechanical energy during driving.

Radio Frequency Identification Technology (Ratio Frequency Identification, RFID) ${ }^{[2]}$ is a non-contact automatic identification technology, its principle is using the RF signal and space coupling transmission characteristics, inductive coupling which is based on the law of electromagnetic induction, and electromagnetic coupling is based on the principle of radar model, 
the emitted electromagnetic wave hits the target and reflect back the target information, based on the spatial propagation of electromagnetic waves. Radio frequency identification system is generally composed of two parts, namely, electronic tags (Tag) and readers (Reader). In the TPMS, tag is attached to the telemetry module and the Reader is completed by the receiving module.

\section{Summary}

With the continuous development of modern sensor technology, the structure of TPMS constantly changes. Each type has different applications. In this paper, A principle is raised to classify the existing TPMS, given to more comprehensive discussion of the characteristics and differences between various types of TPMS.

\section{References}

[1] Liu Fengjun. Li Haifang; Exploration and improvement of electromechanical TPMS sensor; Auto Electric.2004.5

[2] You Zhanqing; Radio frequency identification (RFID) theory and application, Beijing;

Electronic Industry Press, 2004

[3] Qiu Bin; Analysis of tire pressure monitoring system and domestic development suggestion; special purpose vehicle.2006.8

[4] Tan Zefei, He Chao; the principle and application of a tire pressure monitoring system; Journal of Southwest Forestry University, 2005.25 